\title{
Measuring the spectral running from cosmic microwave background and primordial black holes
}

\author{
Jun $\mathbf{L i}^{1,2, a}$, Qing-Guo Huang ${ }^{1,2,3,4, b}$ \\ ${ }^{1}$ CAS Key Laboratory of Theoretical Physics, Institute of Theoretical Physics, Chinese Academy of Sciences, Beijing 100190, China \\ ${ }^{2}$ School of Physical Sciences, University of Chinese Academy of Sciences, No. 19A Yuquan Road, Beijing 100049, China \\ ${ }^{3}$ Center for Gravitation and Cosmology, College of Physical Science and Technology, Yangzhou University, Yangzhou 225009, China \\ ${ }^{4}$ Synergetic Innovation Center for Quantum Effects and Applications, Hunan Normal University, Changsha 410081, China
}

Received: 11 August 2018 / Accepted: 20 November 2018 / Published online: 27 November 2018

(C) The Author(s) 2018

\begin{abstract}
We constrain the spectral running by combining cosmic microwave background (CMB) data, baryon acoustic oscillation (BAO) data and the constraint from primordial black holes (PBHs). We find that the constraint from PBHs has a significant impact on the running of running of scalar spectral index, and a power-law scalar power spectrum without running is consistent with observational data once the constraint from PBHs is taken into account. In addition, from the constraints on the slow-roll parameters, the derived tensor spectral index in the single-field slow-roll inflation model is quite small, namely $\left|n_{t}\right| \lesssim 9.3 \times 10^{-3}$ which will be very difficult to be measured by CMB data only in the future, and the absolute value of derived running of tensor spectral index is not larger than $2.1 \times 10^{-4}$ at $95 \%$ confidence level.
\end{abstract}

\section{Introduction}

The current cosmological observations, for example the Planck satellite $[1,2]$, provide strong evidence for the standard six-parameter $\Lambda \mathrm{CDM}$ model. However, when the base model is extended and other cosmological parameters are allowed to freely vary, a few anomalies are present. For example, the running of running of the scalar spectral index which is defined by

$\beta_{s} \equiv \frac{d^{2} n_{s}}{d \ln k^{2}}$

reads

$\beta_{s}=0.025 \pm 0.013$

\footnotetext{
a e-mail: lijun@itp.ac.cn

b e-mail: huangqg@itp.ac.cn
}

from Planck data in Ref. [2] and

$\beta_{s}=0.021 \pm 0.013$

from the combination of Planck [2], BK14 [3] and Baryon Acoustic Oscillation (BAO) [4-6] data in Ref. [7] at 68\% confidence level (CL) on the cosmic scales, where $n_{s}$ is the spectral index of scalar power spectrum.

In 1971, Hawking proposed that the highly overdense region of inhomogeneities would eventually cease expanding and collapse into a black hole in [8]. A large positive value of the running of running may lead to the formation of primordial black holes (PBH) at small scales in the early universe. Different from the astrophysical black holes which should be heavier than a particular mass (around 3 solar mass $M_{\odot}$ ), PBHs can have very small masses. However, the PBHs with masses smaller than $10^{-18} M_{\odot}$ would have completely evaporated by now due to the Hawking radiation. In particular, the quantum emissions from PBHs with mass around $10^{-18} M_{\odot}$ can generate a $\gamma$-ray background which should be observed today. Conversely, the non-detection of such a $\gamma$-ray background in our Universe provides a stringent constraint on the PBHs with such a mass. Recently various constraints on the abundance of PBHs are present in [9-22]. Ones expect that the absence of PBHs should tightly constrain the running of running of scalar spectral index. See some recent related works in $[23,24]$.

On the other hand, inflation [25-28] is taken as the leading paradigm for the physics in the very early universe. The initial inhomogeneities and spatial curvature are supposed to be stretched away by the quasi-exponential expansion of inflation. Because the Hubble parameter is roughly a constant during inflation, it predicts a nearly scale-invariant power spectrum of the curvature (scalar) perturbations seeded by the quantum fluctuations of inflaton field during inflation. Typically, the scalar spectral index $n_{s}$ is related to the number 
of e-folds $N$ before the end of inflation by $n_{s}-1 \sim-1 / N$, and then $\beta_{s} \sim-1 / N^{3} \sim\left(n_{s}-1\right)^{3} \sim-\mathcal{O}\left(10^{-5}\right)$. In this sense, the running of running of scalar spectra index given in Eqs. (2) and (3) seems too large to fit in the typical inflation models.

In this paper we adopt two methods to compare inflationary predictions with current cosmological datasets, in particular including the constraint from the PBHs. The first method consists of a phenomenological parameterization of the primordial spectra of both scalar and tensor perturbations, and the second exploits the analytic slow-roll-parameter dependence of primordial perturbations.

This paper is organized as follows. In Sect. 2, we explain the datasets used in this paper. In Sects. 3 and 4, we present the constraints on the phenomenological parameters and the slow-roll parameters with the publicly available codes CosmoMC [29], respectively. A brief summary will be given in Sect. 5 .

\section{Data}

The full-mission Planck observes the temperature and polarization anisotropies of the cosmic microwave background $(\mathrm{CMB})$ radiation. In this paper we also combine all the data taken by the BICEP2 and Keck Array CMB polarization experiments up to and including the 2014 observing season [3] with the Planck data.

Baryon Acoustic Oscillation (BAO) detections measure the correlation function and power spectrum in the clustering of galaxies. Measuring the position of these oscillations in the matter power spectra at different redshifts removes degeneracies in the interpretation of the CMB anisotropies. The BAO data adopted in this paper include 6dFGS [5], MGS [6], BOSS DR11_Ly $\alpha$ [30], BOSS DR12 with nine anisotropic measurements [31], and eBOSS DR14 [32].

The mass of $\mathrm{PBH}$ is roughly given by the horizon mass at the time of formation, namely

$m=\gamma \frac{4 \pi}{3} \rho H^{-3}$

where $\gamma=3^{-3 / 2} \simeq 0.2$ [33]. In the comoving units, the horizon scale is $R=(a H)^{-1} \propto a$ during radiation domination. Since constant-entropy expansion implies $T \propto g_{*}^{-1 / 3} a^{-1}$, $\rho \propto g_{*} T^{4} \propto g_{*}^{-1 / 3} a^{-4}$ and then the mass of $\mathrm{PBH}$ is $m \propto g_{*}^{-1 / 3} a^{2}$, where the number of relativistic degrees of freedom $g_{*}$ is around 3 at matter-radiation equality and $10^{2}$ in the early universe. Therefore

$\frac{m}{M_{\odot}} \simeq\left(k_{\odot} R\right)^{2}$,

where $k_{\odot} \simeq 10^{6} \mathrm{Mpc}^{-1}$ is the perturbation mode which re-enter the horizon when the one-solar mass $\mathrm{PBH}$ can be formed. On the other hand, due to the quantum effects near the event horizon of black hole, it could emit black body radiation which is the so-called Hawking radiation [34], and is supposed to evaporate completely within the time scale

$T_{\text {eva }}(m) \sim \frac{G^{2} m^{3}}{\hbar c^{4}} \sim 10^{64}\left(\frac{m}{M_{\odot}}\right)^{3} \mathrm{yr}$.

It implies that the PBHs with a mass smaller than $10^{-18} M_{\odot}$ would have evaporated by now, but PBHs with a mass of around $10^{-18} M_{\odot}$ would be producing photons with energy of order $100 \mathrm{MeV}$ and form a $\gamma$-ray background at the present epoch [35]. The observational limits on the existence of an extragalactic $\gamma$-ray background between $30 \mathrm{MeV}$ and $120 \mathrm{GeV}$ were provided by EGRET observations [36] which indicates that the abundance of PBHs with a mass of around $10^{-18} M_{\odot}$ in dark matter should be tiny $[37,38]$. Even though the formation process of the PBHs is still under debates, as a conservative estimation, the scalar power spectrum at the scale $k_{c} \simeq 10^{15} \mathrm{Mpc}^{-1}$ corresponding to the formation of $\mathrm{PBH}$ with a mass of around $10^{-18} M_{\odot}$ should be smaller than unity, namely

$P_{S}\left(k_{c}\right) \leq 1$

otherwise, the abundance of PBHs with a mass of around $10^{-18} M_{\odot}$ in dark matter would be order of one and generate an observable $\gamma$-ray background at the current epoch. Therefore, we take Eq. (7) as the constraint on the scalar power spectrum from $\mathrm{PBHs}$.

Actually Eq. (7) is a very conservative constraint. The ranges of $k$ corresponding to the $\gamma$-ray background extend from $10^{14} \mathrm{Mpc}^{-1}$ to $10^{15} \mathrm{Mpc}^{-1}$, and the upper limit of scalar power spectrum is generally in the range of 0.1 to 0.01 . Therefore a more restricted constraint might be $P_{S}(k=$ $\left.10^{15} \mathrm{Mpc}^{-1}\right)<10^{-2}$ which is denoted by " $\mathrm{PBH}^{*}$ ".

\section{Constraints on the spectral running from CMB and PBHs}

In this section, the power spectra of the scalar and tensor perturbations are parameterized by

$$
\begin{aligned}
& P_{s}(k)=A_{s}\left(\frac{k}{k_{*}}\right)^{n_{s}-1+\frac{1}{2} \alpha_{s} \ln \left(k / k_{*}\right)+\frac{1}{6} \beta_{s}\left(\ln \left(k / k_{*}\right)\right)^{2}+\cdots}, \\
& P_{t}(k)=A_{t}\left(\frac{k}{k_{*}}\right)^{n_{t}+\frac{1}{2} \alpha_{t} \ln \left(k / k_{*}\right)+\cdots},
\end{aligned}
$$

where $A_{s}\left(A_{t}\right)$ is the scalar (tensor) amplitude at the pivot scale $k_{*}=0.05 \mathrm{Mpc}^{-1}, n_{s}$ is the scalar spectral index, $\alpha_{s} \equiv \mathrm{d} n_{s} / \mathrm{d} \ln k$ is the running of scalar spectral index, $\beta_{s} \equiv \mathrm{d}^{2} n_{s} / \mathrm{d} \ln k^{2}$ is the running of running of scalar spectral index, $n_{t}$ is the tensor spectral index, and $\alpha_{t} \equiv \mathrm{d} n_{t} / \mathrm{d} \ln k$ is the running of tensor spectral index. In literature some 
Table 1 The $68 \%$ limits on the cosmological parameters in the $\Lambda \mathrm{CDM}+r+\alpha_{s}+\beta_{s}$ model from the data combinations of $\mathrm{CMB}+\mathrm{BAO}$ and $\mathrm{CMB}+\mathrm{BAO}+\mathrm{PBH}$ respectively

\begin{tabular}{lll}
\hline Parameter & $\mathrm{CMB}+\mathrm{BAO}$ & $\mathrm{CMB}+\mathrm{BAO}+\mathrm{PBH}$ \\
\hline$\Omega_{b} h^{2}$ & $0.02232 \pm 0.00015$ & $0.02238 \pm 0.00014$ \\
$\Omega_{c} h^{2}$ & $0.1180 \pm 0.0008$ & $0.1179 \pm 0.0008$ \\
$100 \theta_{\mathrm{MC}}$ & $1.0410 \pm 0.0003$ & $1.0410 \pm 0.0003$ \\
$\tau$ & $0.079 \pm 0.012$ & $0.073 \pm 0.012$ \\
$\ln \left(10^{10} A_{s}\right)$ & $3.088 \pm 0.023$ & $3.077 \pm 0.022$ \\
$n_{s}$ & $0.9660 \pm 0.0040$ & $0.9693 \pm 0.0038$ \\
$\alpha_{s}$ & $0.0077_{-0.0103}^{+0.0104}$ & $-0.0063_{-0.0072}^{+0.0073}$ \\
$\beta_{s}$ & $0.019 \pm 0.013$ & $-0.0035_{-0.0023}^{+0.0065}$ \\
$r(95 \% \mathrm{CL})$ & $<0.086$ & $<0.079$ \\
\hline
\end{tabular}

inflationary models for PBH formation require some feature in the scalar power spectrum which cannot be modeled by the expansion in Eq. (8), and then our analysis would not be applicable for those cases. Usually we introduce a new parameter, namely the tensor-to-scalar ratio $r$, to quantify the tensor amplitude compared to the scalar amplitude at the pivot scale:

$r \equiv \frac{A_{t}}{A_{s}}$

It is known that the single parameter Harrison-Zeldovich spectrum $\left(n_{s}=1\right)$ does not fit the data and at least the parameters $A_{s}$ and $n_{s}$ in the expansion of the primordial scalar power spectrum are needed. Here the spectral index of tensor power spectrum is set as $n_{t}=-r / 8$ which is nothing but the consistency relation to lowest order in the single-field slow-roll inflation model [39,40], and $\alpha_{t}=0$.

We consider the six parameters in the standard $\Lambda \mathrm{CDM}$ model, i.e. the baryon density parameter $\Omega_{b} h^{2}$, the cold dark matter density $\Omega_{c} h^{2}$, the angular size of the horizon at the last scattering surface $\theta_{\mathrm{MC}}$, the optical depth $\tau$, the scalar amplitude $A_{s}$ and the scalar spectral index $n_{s}$. We extend this scenario by adding the running of the scalar spectral index $\alpha_{s}$, the running of running $\beta_{s}$ and the tensor amplitude, or equivalently the tensor-to-scalar ratio $r$. We constrain all of these 9 parameters in the $\Lambda \mathrm{CDM}+r+\alpha_{s}+\beta_{s}$ model by adopting two different data combinations, namely $\mathrm{CMB}+\mathrm{BAO}$ and $\mathrm{CMB}+\mathrm{BAO}+\mathrm{PBH}$, respectively. The results are given in Table 1 and Fig. 1. The constraint on the running of running of scalar spectral index is

$\beta_{s}=0.019 \pm 0.013$

at $68 \% \mathrm{CL}$ from $\mathrm{CMB}+\mathrm{BAO}$, and

$\beta_{s}=-0.0035_{-0.0023}^{+0.0065}$

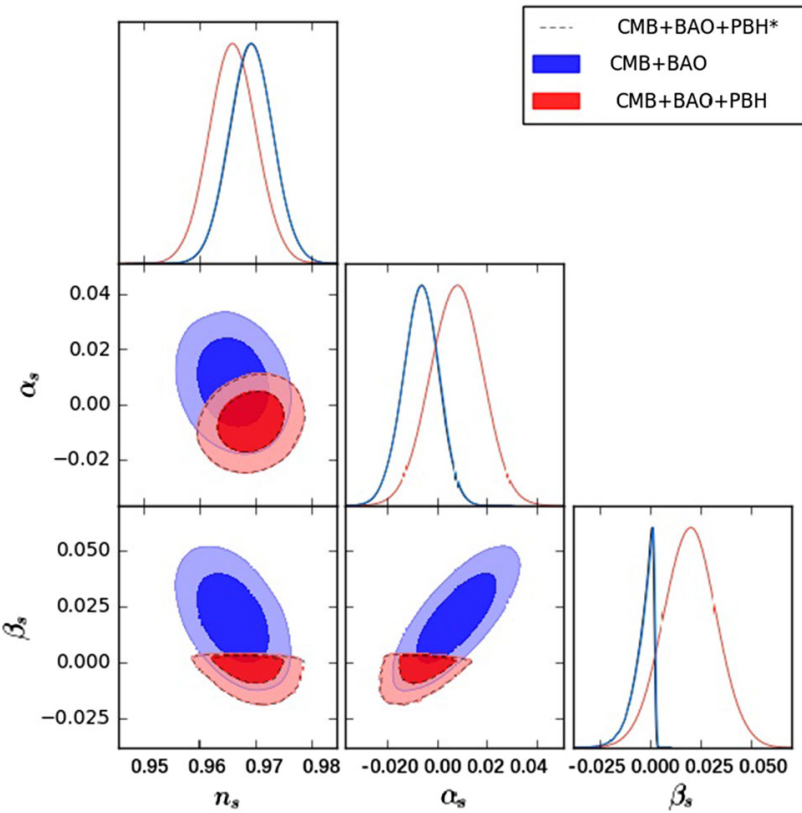

Fig. 1 The contour plots and the likelihood distributions for $n_{s}, \alpha_{s}, \beta_{s}$ at the $68 \%$ and $95 \% \mathrm{CL}$ by using $\mathrm{CMB}+\mathrm{BAO}+\mathrm{PBH}^{*}, \mathrm{CMB}+\mathrm{BAO}$ and $\mathrm{CMB}+\mathrm{BAO}+\mathrm{PBH}$, respectively

at $68 \% \mathrm{CL}$ from $\mathrm{CMB}+\mathrm{BAO}+\mathrm{PBH}$. We see that the constraint on the running of running of scalar spectral index is significantly affected by adding the constraint of PBHs, and a power-law scalar power spectrum without running is consistent with the data within $68 \%$ confidence level once the constraint from PBHs is taken into account. The PBH constraint changes the contours and likelihoods from the blue ones to the red ones because the positive value of $\beta_{s}$ yields a significant growth of scalar power spectrum at small scales which may generate too many PBHs conflicting with the measurement of $\gamma$-ray background. Note that the constraints on the cosmological parameters by adding $\mathrm{PBH}^{*}$ is quite similar to those by adding PBH.

\section{Constraints on the slow-roll parameters}

In this section, we focus on the canonical single-field slowroll inflation model in which the inflation is driven by the inflaton potential $V(\phi)$. The dynamics of inflation is govern by

$$
\begin{aligned}
& H^{2}=\frac{1}{3 M_{p}^{2}}\left[\frac{1}{2} \dot{\phi}^{2}+V(\phi)\right], \\
& \ddot{\phi}+3 H \dot{\phi}+V^{\prime}(\phi)=0,
\end{aligned}
$$

where $M_{p}=1 / \sqrt{8 \pi G}$ is the reduced Planck energy scale and the dot and prime denote the derivative with respective to the cosmic time $t$ and the inflaton field $\phi$, respectively. The 
inflaton field slowly rolls down its potential if $\epsilon \ll 1$ and $|\eta| \ll 1$, where

$\epsilon=\frac{M_{p}^{2}}{2}\left(\frac{V^{\prime}(\phi)}{V(\phi)}\right)^{2}$

$\eta=M_{p}^{2} \frac{V^{\prime \prime}(\phi)}{V(\phi)}$.

The amplitude of scalar and tensor perturbation power spectra are given by, in Ref. [41],

$P_{s} \simeq\left[1+\frac{25-9 c}{6} \epsilon-\frac{13-3 c}{6} \eta\right] \frac{V / M_{p}^{4}}{24 \pi^{2} \epsilon}$,

$P_{t} \simeq\left[1-\frac{1+3 c}{6} \epsilon\right] \frac{V / M_{p}^{4}}{3 \pi^{2} / 2}$,

where $c \simeq 0.08145$, and then

$$
\begin{aligned}
r \approx & 16 \epsilon\left[1-\frac{13-3 c}{6}(2 \epsilon-\eta)\right], \\
n_{t} \approx & -2 \epsilon-\frac{2(2+3 c)}{3} \epsilon^{2}-\frac{1-3 c}{3} \epsilon \eta, \\
\alpha_{t} \approx & -8 \epsilon^{2}+4 \epsilon \eta-\frac{8(5+6 c)}{3} \epsilon^{3}+2(1+7 c) \epsilon^{2} \eta \\
& +2(1-c) \epsilon \eta^{2}, \\
n_{s} \approx & 1-6 \epsilon+2 \eta+\frac{2(22-9 c)}{3} \epsilon^{2}-2(7-2 c) \epsilon \eta \\
& +\frac{2}{3} \eta^{2}, \\
\alpha_{s} \approx & -24 \epsilon^{2}+16 \epsilon \eta-2 \xi+\frac{8(41-18 c)}{3} \epsilon^{3} \\
& -\frac{4(109-36 c)}{3} \epsilon^{2} \eta+4(9-2 c) \epsilon \eta^{2} \\
& +2(11-3 c) \epsilon \xi-\frac{25-3 c}{6} \eta \xi, \\
\beta_{s} \approx & -192 \epsilon^{3}+192 \epsilon^{2} \eta-32 \epsilon \eta^{2}-24 \epsilon \xi+2 \eta \xi+2 \sigma \\
& +96(13-6 c) \epsilon^{4}-\frac{8(791-288 c)}{3} \epsilon^{3} \eta \\
& +\frac{16(173-48 c)}{3} \epsilon^{2} \eta^{2}-\frac{8(31-6 c)}{3} \epsilon \eta^{3} \\
& +\frac{4(235-72 c)}{3} \epsilon^{2} \xi-\frac{511-111 c}{3} \epsilon \eta \xi \\
& +\frac{29-3 c}{6} \eta^{2} \xi+\frac{25-3 c}{6} \xi^{2}-\frac{103-27 c}{3} \epsilon \sigma \\
& +\frac{55-9 c}{6} \eta \sigma,
\end{aligned}
$$

where

$\xi=M_{p}^{4} \frac{V^{\prime}(\phi) V^{\prime \prime \prime}(\phi)}{V^{2}(\phi)}$,

$\sigma=M_{p}^{6} \frac{V^{\prime 2}(\phi) V^{\prime \prime \prime \prime}(\phi)}{V^{3}(\phi)}$.

See some related references in [42-44].
Table 2 The 95\% limits on the slow-roll parameters and the derived parameters from the data combinations of $\mathrm{CMB}+\mathrm{BAO}$ and $\mathrm{CMB}+\mathrm{BAO}+\mathrm{PBH}$ respectively

\begin{tabular}{lll}
\hline Parameter & $\mathrm{CMB}+\mathrm{BAO}$ & $\mathrm{CMB}+\mathrm{BAO}+\mathrm{PBH}$ \\
\hline$\epsilon$ & $<0.0053$ & $<0.0047$ \\
$\eta$ & $-0.0112_{-0.0116}^{+0.0122}$ & $-0.0104_{-0.0102}^{+0.011}$ \\
$\xi$ & $-0.0043_{-0.0107}^{+0.0106}$ & $0.0031_{-0.0074}^{+0.0077}$ \\
$\sigma$ & $0.0104_{-0.0133}^{+0.0134}$ & $-0.0018_{-0.0062}^{+0.0045}$ \\
$r$ & $<0.082$ & $<0.073$ \\
$-n_{t}\left(\times 10^{-2}\right)$ & $<1.1$ & $<0.93$ \\
$-\alpha_{t}\left(\times 10^{-4}\right)$ & $<2.6$ & $<2.1$ \\
\hline
\end{tabular}

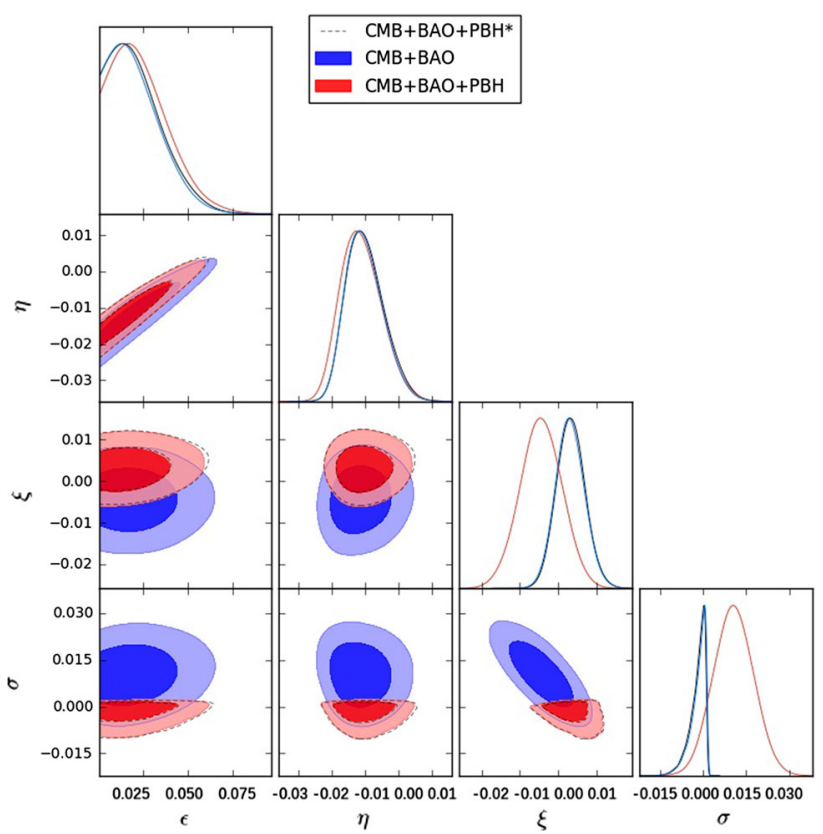

Fig. 2 The contour plots and the likelihood distributions for the slow-roll parameters $\epsilon, \eta, \xi$ and $\sigma$ at the $68 \%$ and $95 \% \mathrm{CL}$ from $\mathrm{CMB}+\mathrm{BAO}+\mathrm{PBH}^{*}, \mathrm{CMB}+\mathrm{BAO}$ and $\mathrm{CMB}+\mathrm{BAO}+\mathrm{PBH}$, respectively

Here the nine parameters sampled in the CosmoMC are $\left\{\Omega_{b} h^{2}, \Omega_{c} h^{2}, \tau, \theta_{\mathrm{MC}}, A_{s}, \epsilon, \eta, \xi, \sigma\right\}$, and then $n_{s}, \alpha_{s}, \beta_{s}, r$, $n_{t}$ and $\alpha_{t}$ are all taken as the derived parameters. The constraints on the slow-roll parameters $\{\epsilon, \eta, \xi, \sigma\}$ and the contour plots of these slow-roll parameters are illustrated in Table 2 and Fig. 2. We see that the constraint on the fourth slow-roll parameter $\sigma$ is improved once the constraint from PBHs is included because the running of running is dominated by it in the slow-roll inflation model.

Here we also work out the predictions of the slow-roll inflation model constrained by the observational data. The parameters $\left\{r, n_{t}, \alpha_{t}\right\}$ characterizing the tensor power spectrum can be obtained by adopting the Eqs. (19), (20) and (21). The results are showed in Table 2 and Fig. 3. 


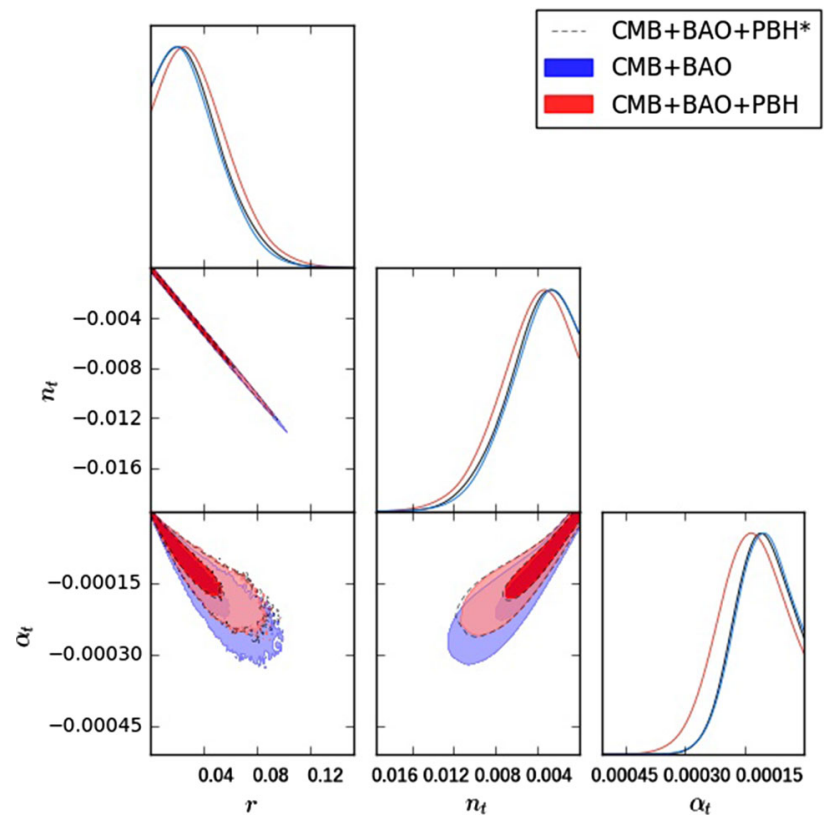

Fig. 3 The derived parameters $r, n_{t}$ and $\alpha_{t}$ for the constrained canonical single-field slow-roll inflation model at the $68 \%$ and $95 \% \mathrm{CL}$ from $\mathrm{CMB}+\mathrm{BAO}+\mathrm{PBH}^{*}, \mathrm{CMB}+\mathrm{BAO}$ or $\mathrm{CMB}+\mathrm{BAO}+\mathrm{PBH}$

In particular, we notice that both the derived tensor spectral index $n_{t}$ and its running $\alpha_{t}$ are negative in the constrained single-field inflation model, and the constraint on them at 95\% CL are

$$
\begin{aligned}
& -n_{t}<1.1 \times 10^{-2} \\
& -\alpha_{t}<2.6 \times 10^{-4}
\end{aligned}
$$

from $\mathrm{CMB}+\mathrm{BAO}$, and

$$
\begin{aligned}
& -n_{t}<9.3 \times 10^{-3} \\
& -\alpha_{t}<2.1 \times 10^{-4}
\end{aligned}
$$

from $\mathrm{CMB}+\mathrm{BAO}+\mathrm{PBH}$. However, in Ref. [45], the optimistic estimation indicates that the uncertainty at $68 \% \mathrm{CL}$ on the tensor spectral index is $\sigma_{n_{t}} \simeq 1.1 \times 10^{-2}$ due to the cosmic variance only for CMB multipoles less than 300. Because the small-scale CMB B-modes are dominated by CMB lensing, it implies that it is very difficult to measure the tensor spectral index for the single-field slow-roll inflation model by only using the CMB data.

\section{Summary}

In this paper we use two methods to constrain the slow-roll inflation models by combining CMB, BAO and the constraint from PBHs. Even though a positive running of running of scalar spectral index is slightly preferred by the data combination of CMB and BAO datasets, a power-law scalar power spectrum without running is consistent with the data once the constraint from PBHs is taken into account.

We can also directly constrain the slow-roll parameters from the observational data. An advantage of this method is that we can work out the predictions of single-field slow-roll inflation model by using these constrained slow-roll parameters. For example, we illustrate the predictions of the parameters characterizing the tensor power spectrum, and find that both the tensor spectral index and its running are negative and their absolute values are not larger than $9.3 \times 10^{-3}$ and $2.1 \times 10^{-4}$ at $95 \% \mathrm{CL}$, respectively. Our results imply that it is very difficult to measure these two parameters in the future.

Acknowledgements We thank the anonymous referee for valuable suggestions and comments. We acknowledge the use of HPC Cluster of ITP-CAS. This work is supported by grants from NSFC (Grant nos. 11335012, 11575271, 11690021, 11747601), Top-Notch Young Talents Program of China, and partly supported by the Strategic Priority Research Program of CAS and Key Research Program of Frontier Sciences of CAS.

Open Access This article is distributed under the terms of the Creative Commons Attribution 4.0 International License (http://creativecomm ons.org/licenses/by/4.0/), which permits unrestricted use, distribution, and reproduction in any medium, provided you give appropriate credit to the original author(s) and the source, provide a link to the Creative Commons license, and indicate if changes were made. Funded by SCOAP S $^{3}$

\section{References}

1. P.A.R. Ade et al., [Planck Collaboration], Astron. Astrophys. 594, A13 (2016). arXiv:1502.01589 [astro-ph.CO]

2. P.A.R. Ade et al., [Planck Collaboration], Astron. Astrophys. 594, A20 (2016). arXiv:1502.02114 [astro-ph.CO]

3. P.A.R. Ade et al., [BICEP 2 and Keck Array Collaborations], Phys. Rev. Lett. 116, 031302 (2016). arXiv:1510.09217 [astro-ph.CO]

4. H. Gil-Marin, Mon. Not. R. Astron. Soc. 460(4), 4210 (2016). arXiv:1509.06373 [astro-ph.CO]

5. F. Beutler, Mon. Not. R. Astron. Soc. 416, 3017 (2011). arXiv:1106.3366 [astro-ph.CO]

6. A.J. Ross, L. Samushia, C. Howlett, W.J. Percival, A. Burden, M. Manera, Mon. Not. R. Astron. Soc. 449(1), 835 (2015). arXiv: 1409.3242

7. Q.G. Huang, K. Wang, S. Wang, Phys. Rev. D 93(10), 103516 (2016). arXiv:1512.07769 [astro-ph.CO]

8. S. Hawking, Mon. Not. R. Astron. Soc. 152, 75 (1971)

9. L. Chen, Q.G. Huang, K. Wang, JCAP 1612(12), 044 (2016). arXiv: 1608.02174 [astro-ph.CO]

10. A.M. Green, Phys. Rev. D 94(6), 063530 (2016). arXiv:1609.01143 [astro-ph.CO]

11. K. Schutz, A. Liu, Phys. Rev. D 95(2), 023002 (2017). arXiv:1610.04234 astro-ph.CO]

12. S. Wang, Y.F. Wang, Q.G. Huang, T.G.F. Li, Phys. Rev. Lett. 120(19), 191102 (2018). arXiv:1610.08725 [astro-ph.CO]

13. D. Gaggero, G. Bertone, F. Calore, R.M.T. Connors, M. Lovell, S. Markoff, E. Storm, Phys. Rev. Lett. 118(24), 241101 (2017). arXiv:1612.00457 [astro-ph.HE] 
14. Y. Ali-Hamoud, M. Kamionkowski, Phys. Rev. D 95(4), 043534 (2017). arXiv:1612.05644 [astro-ph.CO]

15. D. Aloni, K. Blum, R. Flauger, JCAP 1705(05), 017 (2017). arXiv:1612.06811 [astro-ph.CO]

16. B. Horowitz, arXiv:1612.07264 [astro-ph.CO]

17. F. Kuhnel, K. Freese, Phys. Rev. D 95(8), 083508 (2017). arXiv:1701.07223 [astro-ph.CO]

18. Y. Inoue, A. Kusenko, JCAP 1710(10), 034 (2017). arXiv:1705.00791 [astro-ph.CO]

19. B. Carr, M. Raidal, T. Tenkanen, V. Vaskonen, H. Veermae, Phys. Rev. D 96(2), 023514 (2017). arXiv:1705.05567 [astro-ph.CO]

20. A.M. Green, Phys. Rev. D 96(4), 043020 (2017). arXiv:1705.10818 [astro-ph.CO]

21. M. Zumalacarregui, U. Seljak, arXiv:1712.02240 [astro-ph.CO]

22. Z.C. Chen, Q.G. Huang, arXiv:1801.10327 [astro-ph.CO]

23. B. Carr, T. Tenkanen, V. Vaskonen, Phys. Rev. D 96(6), 063507 (2017). arXiv:1706.03746 [astro-ph.CO]

24. K. Kohri, T. Terada, arXiv:1802.06785 [astro-ph.CO]

25. A.H. Guth, Phys. Rev. D 23, 347 (1981)

26. A.A. Starobinsky, Phys. Lett. B 91, 99 (1980)

27. A.D. Linde, Phys. Lett. 108B, 389 (1982)

28. A. Albrecht, P.J. Steinhardt, Phys. Rev. Lett. 48, 1220 (1982)

29. A. Lewis, S. Bridle, Phys. Rev. D 66, 103511 (2002). astro$\mathrm{ph} / 0205436$

30. T. Delubac et al., BOSS Collaboration, Astron. Astrophys. 574, A59 (2015). arXiv:1404.1801 [astro-ph.CO]
31. Y. Wang et al., [BOSS Collaboration], Mon. Not. Roy. Astron. Soc 469(3), 3762 (2017). arXiv:1607.03154 [astro-ph.CO]

32. M. Ata, Mon. Not. R. Astron. Soc. 473(4), 4773 (2018). arXiv:1705.06373 [astro-ph.CO]

33. B.J. Carr, Astrophys. J. 201, 1 (1975)

34. S.W. Hawking, Commun. Math. Phys. 43, 199 (1975)

35. D.N. Page, S.W. Hawking, Astrophys. J. 206, 1 (1976)

36. P. Sreekumar et al., [EGRET Collaboration], Astrophys. J. 494, 523 (1998). arXiv:astro-ph/9709257

37. B.J. Carr, K. Kohri, Y. Sendouda, J. Yokoyama, Phys. Rev. D 81 , 104019 (2010). arXiv:0912.5297 [astro-ph.CO]

38. B. Carr, F. Kuhnel, M. Sandstad, Phys. Rev. D 94(8), 083504 (2016). arXiv:1607.06077 [astro-ph.CO]

39. A.R. Liddle, D.H. Lyth, Phys. Lett. B 291, 391 (1992). astro$\mathrm{ph} / 9208007$

40. E.J. Copeland, E.W. Kolb, A.R. Liddle, J.E. Lidsey, Phys. Rev. Lett. 71, 219 (1993). hep-ph/9304228

41. Q.G. Huang, Sci. China Phys. Mech. Astron. 58, 11 (2015). arXiv: 1407.4639 [astro-ph.CO]

42. E.D. Stewart, D.H. Lyth, Phys. Lett. B 302, 171 (1993). grqc/9302019

43. S.M. Leach, A.R. Liddle, J. Martin, D.J. Schwarz, Phys. Rev. D 66, 023515 (2002). https://doi.org/10.1103/PhysRevD.66.023515. arXiv:astro-ph/0202094

44. Q.G. Huang, Phys. Rev. D 76, 043505 (2007). astro-ph/0610924

45. Q.G. Huang, S. Wang, arXiv:1701.06115 [astro-ph.CO] 